\title{
OS PICRITOS ALCALINOS DA REGIÃO DE IPORÁ: IMPLICAÇÕES NA GÊNESE DOS COMPLEXOS DO TIPO CENTRAL DO SUL DE GOIÁS
}

\author{
JOSÉ C.M. DANNI*
}

\begin{abstract}
THE ALKALINE PICRITES OF THE IPORÁ REGION: IMPLICATIONS FOR THE SOUTHERN GOIÁS CENTRAL TYPE COMPLEX GÉNESIS. In the Iporá region, Goiás State, the occurrence of several subvolcanic intrusions (sills, dykes and plugs) of alkaline picrites are recognized as part of the Upper Cretaceous Alkaline Province. The picrites have cumulate porphiritic texture, and were formed by 10 to $25 \%$ (vol) of olivine ( $\left.\mathrm{Fog}_{\mathrm{gg}}\right)$ macrocrystals and some clinopyroxenes $(\mathrm{O}$ to $5 \%)$, set in a microcristalline to quenched matrix formed by olivine, diopside, plagioclase, ulvospinel, K-feldspar, nepheline, Ti-biotite and leucite (altered to analcite). Chemically these rocks are ultrabasic alkaline, subsaturated, with ol and ne in the norm. They present $\mathrm{MgO} / \mathrm{MgO}+\mathrm{FeO}$ ratios (molecular \%) between 0.72 and 0.80 , and high values of $\mathrm{Cr}, \mathrm{Ni}, \mathrm{V}$, $\mathrm{Ba}, \mathrm{Sr}$, and light REE. Variations of the major and trace elements are controlled by olivine fractionation. Calculation by mass subtraction of $15-20 \%$ of olivine from the picrites results in Mg-basalt compositions with $\mathrm{MgO}=13 \%$ and $\mathrm{MgO} / \mathrm{MgO}+\mathrm{FeO}$ ratio of 0.71 , that may represent the parental magma from wich the alkaline central complexes of the Iporá region fractionated.
\end{abstract}

Keywords: Alkaline picrites, Petrology, Cretaceous, Goiás.

\begin{abstract}
RESUMO Na região de Iporá, é reconhecida a ocorrência de intrusões subvulcânicas - sills, diques $c$ plugs - formadas por picritos alcalinos de idade cretácica superior. Estas rochas apresentam textura porfirítica cumulativa, formada por 10 a $25 \%$ (vol) de macrocristais de olivina $\left(\mathrm{Fo}_{8 \mathrm{~g}}\right)$ e, às vezes, por clinopiroxênios $(\mathrm{O}$ a $5 \%$ ), imersos em matriz microcristalina composta por olivina, diopsídio, plagioclásio, feldspato potássico, Tibiotita, nefelina e, às vezes, por leucita analcitizada. Possuem caráter ultrabásico alcalino, contendo ol e ne normativas, razões $\mathrm{MgO} / \mathrm{MgO} \pm \mathrm{FeO}$ entre 0,72 e 0,80 e altas concentrações em $\mathrm{Cr}, \mathrm{Ni}, \mathrm{V}, \mathrm{Ba}$, Sr, e ETR leves. As variações químicas entre seus elementos maiores e traços mostram relações controladas, principalmente, pela cristalização fracionada da olivina. Cálculos de massa por subtração dos macrocristais de olivina produzem composições magnesianas $(13 \%$ de $\mathrm{MgO})$ e razões $\mathrm{MgO} / \mathrm{MgO}+\mathrm{FeO}=0,71$. Tais composições podem corresponder ao magma parental gerador dos complexos do tipo central que ocorrem na região.
\end{abstract}

Palavras-chave: Picritos alcalinos, Petrologia, Cretáceo, Goiás.

INTRODUÇÃO Basaltos picríticos (ou simplesmente picritos) são rochas vulcânicas máficas ricas em $\mathrm{MgO}$ (> $9 \%$ ), usualmente portadoras de macrocristais de olivina, e com plagioclásio normativo (an $>50 \%$ ). Com base no grau de saturação, podem ser subdivididos em picritos tholeiíticos (ol-hy normativos) e picritos alcalinos (ne-normativos). Rochas desta natureza têm sido reconhecidas como integrantes essenciais de pilhas vulcânicas tanto em ambientes continentais (Bristow 1984, Cox et al 1984, Cox 1987), como em ilhas oceânicas (Wilkinson \& Hensel 1988, Wilson 1989).

No sul da África, as sequências vulcânicas do sistema Karoo apresentam mais de $4.000 \mathrm{~m}$ de espessura de lavas, contendo $9 \%$ a $24 \%$ de $\mathrm{MgO}$ (Bristow 1984), sendo particularmente abundantes as fácies afíricas e vítreas, com 12 $18 \% \mathrm{MgO}$. Segundo Cox et al. (1984), estas rochas representam apropriadamente composições de líquidos magnesianos primários. Em outras regiões, como por exemplo na costa W da Groenlândia, basaltos picríticos com $15 \%$ a $20 \%$ $\mathrm{MgO}$, de idade Terciária, formam uma pilha vulcânica com mais de $5 \mathrm{~km}$ de espessura (Clarke \& O'Hara 1979). A existência de enormes volumes destas rochas vulcânicas, e ao mesmo tempo a ausência, nas províncias citadas, de rochas mais evoluídas, constituem evidências das mais importantes para considerar estes picritos como representantes de magmas primários contrariamente à proposta que os considera como derivados de frações basálticas enriquecidas em cristais cumulus de olivina.

Em outros domínios geotectônicos, como por exemplo nas ilhas oceânicas de magmatismo intra-placa, a controvérsia relacionada com a origem das rochas picríticas (Nicholls $\&$ Stout 1988) está também centrada sobre esta questão. Os picritos dos vulcões Mauna Loa e Kilauea são considerados como derivados por fracionamento de olivina a baixa pressão, a partir de líquidos magnesianos com $12-14 \% \mathrm{MgO}$ (Wilkinson \& Hensel 1988).

Nos domínios da Bacia do Paraná, apesar da existência de grande volume de rochas basálticas, com espessuras que atingem até $1.500 \mathrm{~m}$ e volume de cerca de $800.000 \mathrm{~km}^{3}$ (Piccirillo \& Melfi 1988), a contribuição de rochas picríticas é aparentemente pouco expressiva, sendo exemplificada por raras ocorrências (ex., Lomba Grande, RS, Viero 1992). Por outro lado, Fodor et al. (1989) têm proposto, para os basaltos tholeiíticos da Bacia do Paraná, origem por fracionamento de olivina, piroxênio e plagioclásio, em condições subcrustais, a partir de magmas picríticos. Piccirillo et al (1988a, b) demonstraram que os basaltos tholeííticos de alto e baixo Ti podem derivar diretamente de manto do tipo granada lherzolito, através de diferentes taxas de fusão, e subsequente contaminação crustal. Apesar disso, admitem a formação de basaltos picríticos previamente à formação dos tholeiítos, durante o estágio pré-rift da evolução global do magmatismo.

O magma parental, que deu origem aos complexos alcalinos zonados da regiẫo de Iporá (Danni 1974), formou por fracionamento uma série representada pela associação dunito-wehrlito-clinopiroxenito-gabro alcalino e nefelina sienito. Em razão da natureza e proporções volumétricas dos produtos fracionados nestes complexos, assim como pelo alto teor de Fo e En das primeiras fases cristalizadas, foi proposto para o líquido original composição do tipo picrito alcalino (Danni 1974, 1976). Neste estudo, relata-se o descobrimento de diversos focos subvulcânicos de picritos al- 
calinos nessa região, o que vem fornecer evidências adicionais para a origem daqueles complexos, a partir de líquidos picríticos, como também traz novos dados sobre a incidência desse tipo de magmatismo nos domínios setentrionais da Bacia do Paraná, durante o Cretáceo Superior.

QUADRO GEOLÓGICO REGIONAL As regiões situadas ao longo da margem setentrional da Bacia do Paraná (p.ex., Rio Verde - Iporá) foram afetadas por importantes eventos de magmatismo alcalino durante o Cretáceo Superior. Nessas áreas, ocorrem diversos exemplos de atiyidade magmática, sendo particularmente importantes as intrusões zonadas do tipo central (Guimarães et al 1968, Danni 1974, Barbour 1976, Faria et al 1970, Pena \& Figueiredo 1972) e um grande número de intrusões subvulcânicas, exemplificadas por sills, diques, plugs e pipes, além de ocorrência de campos de lavas e de depósitos piroclásticos associados (Hasui et al 1971, Gaspar \& Danni 1981, Danni 1985, 1990, Danni et al. 1990). A esta diversidade de formas soma-se a recorrência, num mesmo espaço geológico, de pulsos magmáticos com dinamismos e composições variados.

Neste panorama regional, a área situda a NW de Iporá foi sede de diversas intrusões subvulcânicas, constituídas principalmente por picritos alcalinos, os quais são objeto do presente estudo (Fig. 1).

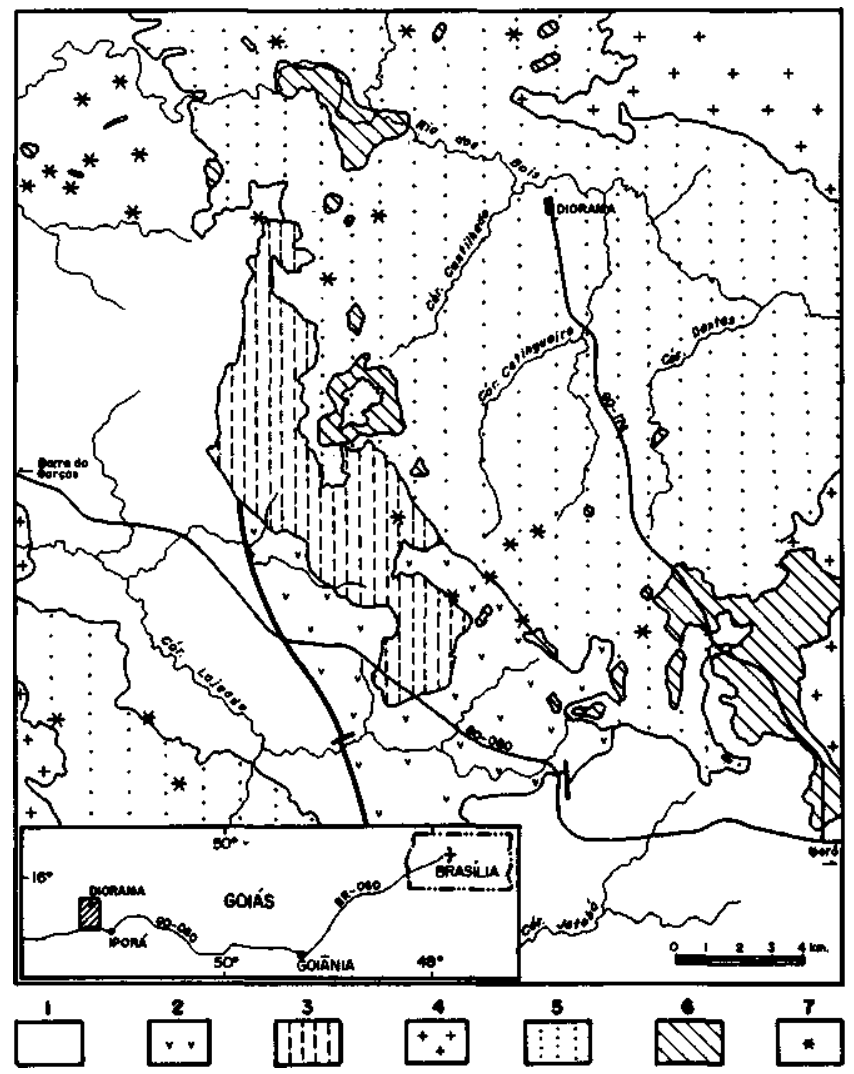

Figura 1 - Mapa geológico da área estudada. 1. Gnaisses graníticos-granodioríticos miloníticos; 2. Sequência vulcano-sedimentar do tipo arco de ilha; 3. Intrusão gabrodiorítica; 4. Granitos sin e pós-colisionais; 5. Formações sedimentares siluro-devonianas; 6. Intrusões alcalinas gabro-sieníticas e traquíticas; 7. ocorrências de picritos alcalinos e rochas afins

Rgurc 1 - Geologic map of the área. 1. Mylonitic granite-grano dioritic gneisses; 2. Island are volcano-sedimentary type sequence; 3. Gabbro-dioritic intrusion; 4. Syn and post-collisional granites; 5. Silurian and Devonian sedimentary formations; 6. Alkaline gabbro-syenitic and trachytic intrusions; 7. occurrences of alkali-picrites and related rocks
Genericamente, as rochas que constituem o embasamento da Bacia do Paraná, encaixantes dos picritos, compreendem os terrenos graníticos e vulcano-sedimentares do Proterozóico Superior (Pimentel \& Fuck 1987). Amplas zonas de cisalhamento, de direção N10-20W, seccionam estes terrenos e condicionam os limites de complexos graníticos sin e pós-orogênicos.

$\mathrm{Na}$ área, a cobertura sedimentar da Bacia do Paraná perfaz cerca de $200 \mathrm{~m}$ de espessura, abrangendo as formações siluro-devonianas (Vila Maria, Furnas e Ponta Grossa). Essas formações ocorrem como restos erosivos, constituindo tabuleiros de arenitos, ou como compartimentos rebaixados no interior de blocos tectônicos, delimitados por falhas gravitacionais com direções N30-40W e N50E. Tais linhas tectônicas foram especialmente ativas durante o Cretáceo Superior, tendo então possibilitado o acesso e a instalação do magmatismo alcalino.

INSTRUÇÕES SUBVULCÂNICAS PICRÍTICAS Três principais formas de intrusões são comumente encontradas na área: diques, plugs e sills. Os diques ocorrem preferencialmente alojados nos terrenos Pré-Cambrianos e formam intrusões com poucos metros de largura, atingindo algumas dezenas de metros de comprimento, preenchendo preferencialmente as fraturas com direções N30W e N50E.

Os sills ocorrem associados às unidades sedimentares, sendo particularmente frequentes no interior da Formação Vila Maria, embora também ocorram entre arenitos da Formação Ponta Grossa. Normalmente, apresentam espessuras inferiores a $5 \mathrm{~m}$ e, lateralmente, podem estender-se por mais de $500 \mathrm{~m}$. Esses corpos são bastante homogéneos, sem apresentar internamente zonas com composições e texturas contrastadas. Em particular, não foram observadas fácies de acumulação de olivina e/ou piroxênios, próximo a base dessas soleiras. A inexistência dessas fácies, assim como a frequente ocorrência de texturas quenched nos picritos indicam um processo de cristalização por super-resfriamento, fato que parece ter inibido a diferenciação in situ dos corpos picríticos.

Os plugs constituem intrusões cilíndricas, com diâmetros que podem ultrapassar $200 \mathrm{~m}$, como bem exemplificam as ocorrências que afloram nos altos da Serra de Sto. António.

PETROGRAFIA Nos diversos tipos e locais de ocorrência, os picritos apresentam invariavelmente texturas porfiríticas, formadas por abundantes macrocristais (1-5 mm) de olivinas e mais raramente de clinopiroxênio.

Os macrocristais de olivina correspondem a fenocristais $(0,5-3 \mathrm{~mm})$ euédricos, apresentando faces de prismas e domos bem desenvolvidos e a xenocristais $(2-5 \mathrm{~mm})$, com formas ovóides e margens corroídas, apresentando em seus interiores bandas de deformação produzidas a altas temperaturas. Nessas rochas, são também comuns as ocorrências de xenólitos ( $2-8 \mathrm{~mm}$ ) de composição dunítica (olivina adcumulado) e mais raramente formados por agregados de augita e olivina (= olivina clinopiroxenitos).

Quando presentes, os fenocristais de augita formam prismas com até $1 \mathrm{~cm}$ de comprimento, fortemente zonados e com margens do tipo Ti-augita.

As fácies que contêm maior quantidade de macrocristais de olivina $(20$ - $25 \%$ vol.) são claramente cumulíticas, sendo derivadas do fracionamento e acumulação de olivina, em condições aparentemente subvulcânicas. Por outro lado, parte dos macrocristais de olivina, especialmente aqueles que apresentam estruturas internas produzidas por deformações, podem resukar da cominuição de cumulados de mais alta pressão, derivados por fracionamento polibário de líquidos picríticos ou até mesmo representar restitos mantélicos.

De modo geral, a matriz dessas rochas é microcristalina, sendo formada por uma rede de prismas de Ti-augita, 
cristais de ulvoespinélio, plagioclásio e Ti-biotita. Fácies com texturas derivadas de super-resfriamento e frações vitreas são frequentemente encontradas próximo às margens das intrusões.

$\mathrm{Na}$ matriz, a biotita ocorre tanto em cristais isolados como sob a forma de coroas envolvendo espinélio. As porções intersticiais da matriz são formadas por plagioclásio e nefe-lina e, as vezes, por material vítreo. Nas fácies portadores de leucita, esse mineral é mais precoce do que os feldspatos e ocorre como microfenocristais (1,0 a $0,5 \mathrm{~mm})$.

A maioria das fácies estudadas possui minerais de alteração, tais como: serpentina, magnetita, iddingsita, natrolita, analcita, calcedônia e calcita.

Genericamente, os picritos que ocorrem na região de Iporá podem ser classificados como álcali-picritos normais (1.e., portadores de nefelina), biotita álcali-picritos e "leucita" picritos (mais raros). Variações composicionais são observadas notadamente para ankaramitos e olivina-melanefelinitos, como resultado da redução do conteúdo modal de plagioclásio.

QUÍMICA MINERAL As características composicionais das principais fases minerais formadoras dos picritos são a seguir resumidas, estando os dados químicos disponíveis aos interessados com o Autor. As análises químicas foram realizadas no Laboratório de Microssonda Eletrônica do Instituto de Geociências da Universidade de Brasília, com o equipamento CAMEGA SX-50, operando a $15 \mathrm{KV}$ e 20 n A, utilizando padrões sintéticos e naturais.

Olivina: os macro e microcristais apresentam composição muito próximas, apesar das diferentes formas de ocorrência. Correspondem a crisolitas fracamente zonadas com teores $\mathrm{Fo}_{88.86 \%}$, com cerca de $0,50 \%$ de $\mathrm{CaO}$ e $0,30 \%$ de $\mathrm{NiO}$.

Cromita: ocorre em microcristais inclusos em olivinas e correspondem aos tipos magnésio-cromitas aluminosas, composicional e texturalmente semelhantes às encontradas nos peridotitos dos complexos plutônicos de Iporá. Apresentam variações químicas controladas principalmente pela

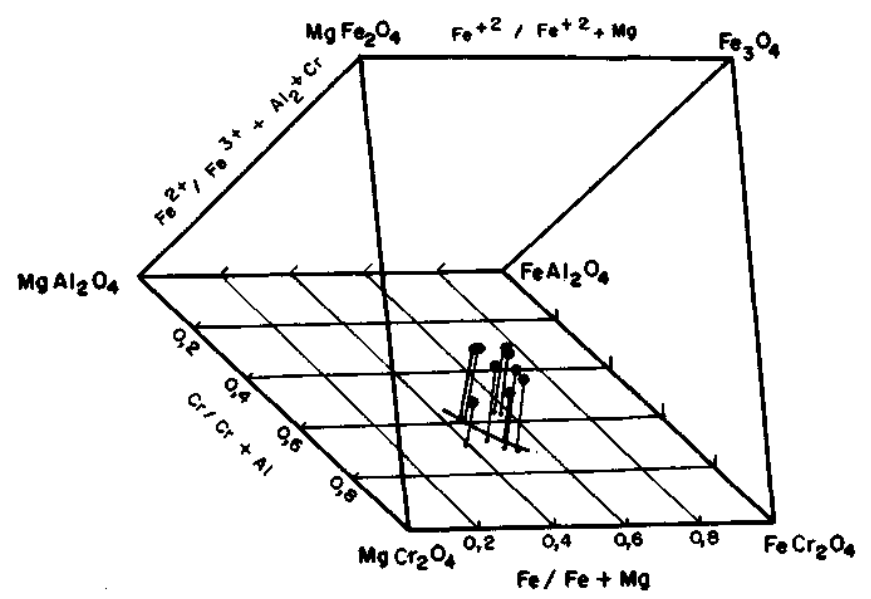

Figura 2 - Variação composicional das cromitas no prisma de classificação dos espinélios

Figure 2 - Compositional variation of the chromites in the spinel classification prism

substituição do $\mathrm{Cr}$ por $\mathrm{Al}$ e do $\mathrm{Mg}$ por Fe (Fig. 2). De acordo com Frost \& Lindsley (1992), a entrada preferencial de Al na composição das cromitas é facilitada pelo grau de insaturação do magma basáltico.

Ulvoespinélio é o óxido de cristalização mais tardia, sendo restrito à matriz e caracteristicamente contém cerca de $22 \%$ da molécula de magnetita, em solução sólida.
$\% \mathrm{SiO}_{2}$

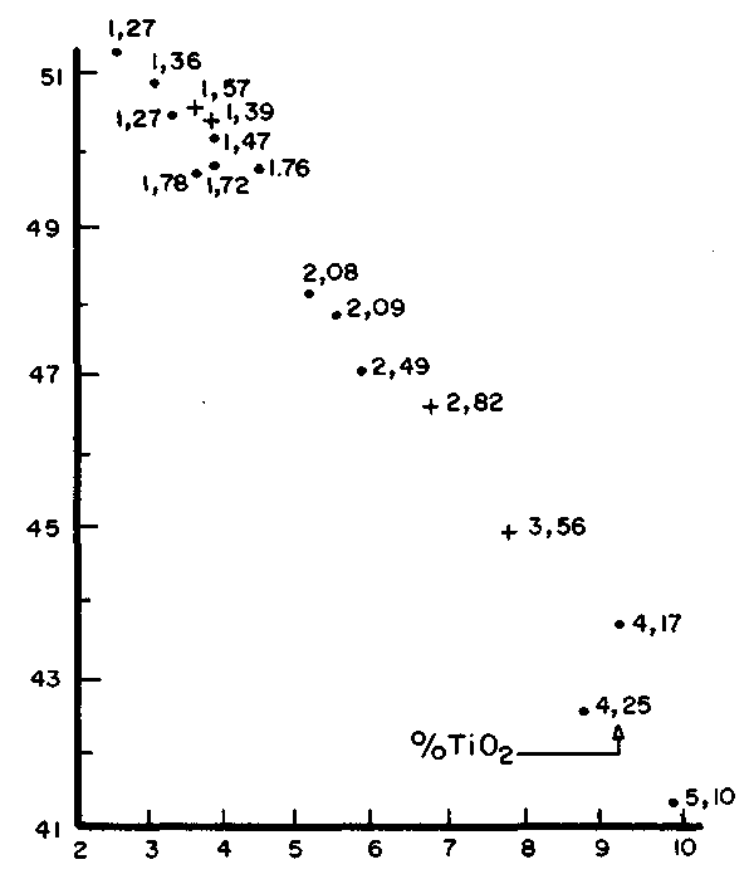

$\% \mathrm{Al}_{2} \mathrm{O}_{3}$

Figura 3 - Variações composicionais dos clinopiroxênios: a substituição de Si por Al (sítio Z) é associada à entrada de $\mathrm{Ti}(Y)$, exemplificando a participação da molécula CaTiAl $\mathrm{O}_{6}$ nestes piroxênios

Figure 3 - Compositional variations of the clinopyroxenes: the substitution of $\mathrm{Si}$ by $\mathrm{Al}$ (in $\mathrm{Z}$ site) can be related to the incorporation of the $\mathrm{CaTiAl}_{2} \mathrm{O}_{6}$ molecule

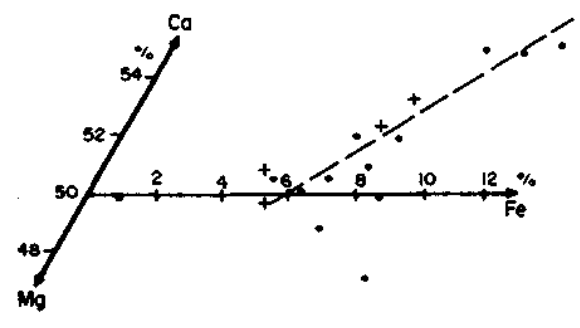

CPX - MATA1Z , FENOCRISTAL

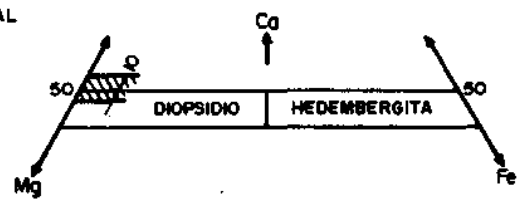

Figura 4 - Posição dos clinopiroxênios no diagrama $\mathrm{Ca}-\mathrm{Mg}-\mathrm{Fe}$

Figure 4 - Clinopyroxene compositions in the $\mathrm{Ca}-\mathrm{Mg}$-Fe diagram

O clinopiroxênio, tanto em fenocristais como matriciais, corresponde a diopsídios com excesso em cálcio, que, quando zonados, evoluem para augitas fassaíticas, em virtude da maior participação de $\mathrm{Al}(\mathrm{Z})$, Ti,Fe e $\mathrm{Ca}$, e consequente empobrecimento em Si e Mg (Figs. 3 e 4).

Em média, os plagioclásios correspondem à composição da andesina rica em potássio $\left(\mathrm{Ab}_{54} \mathrm{An}_{34}, \mathrm{Or}_{12}\right)$ e o feldspato alcalino tem composição média $\mathrm{Or}_{66} \mathrm{Ab}_{29} \mathrm{An}_{5}$.

Os microcristais isométricos de feldspatóides apresentam composições de analcita, com baixo $\mathrm{K}_{2} \mathrm{O}(<0,40 \%)$ e são interpretados como derivados da alteração de leucitas primárias. 


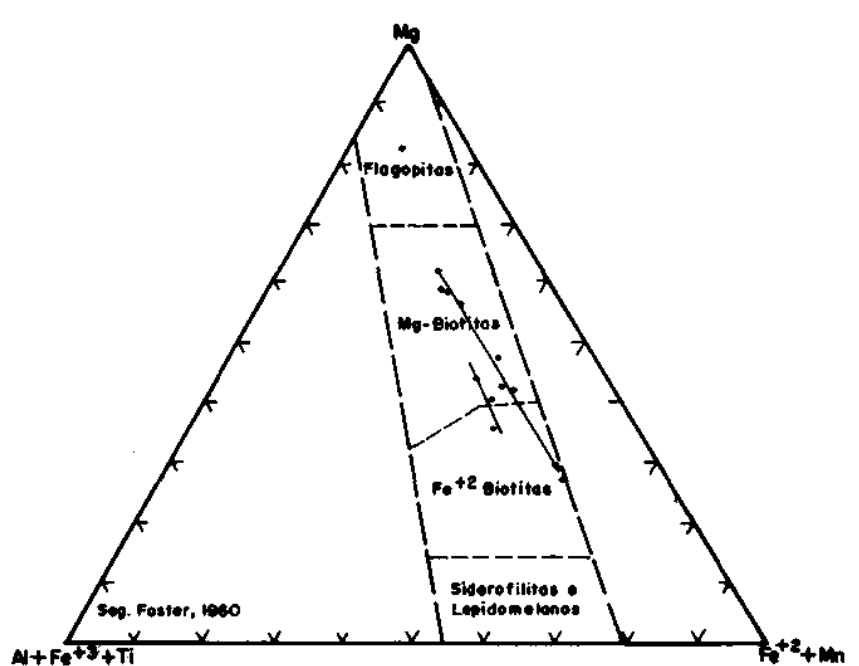

Figura 5 - Diagrama de classificação (Foster 1960) evidenciando as variações químicas das Ti-biotitas apresentadas neste trabalho

Figure 5 - Classification diagram (Foster 1960) displaying the chemical variations of the Ti-biotites from this work

As Ti-biotitas usualmente apresentam zonação composicional, com aumento do teor de Fe para as bordas, e altos teores de $\mathrm{TiO}_{2}(7,0-8,0 \%)$ e baixo $\mathrm{BaO}(2,0 \%)$ (Fig. 5).

CARACTERÍSTICAS QUÍMICAS Os dados sobre a composição química (Tab. 2) dos picritos estudados evidenciam, para essas rochas, natureza ultrabásica-alcalina, subaluminosa, sendo notável o caráter altamente magnesiano ( $\mathrm{MgO}$ entre 16 e 21\%), traduzindo-se em valores de 0,72 a 0,80 para a razão $\mathrm{MgO} / \mathrm{MgO}+\mathrm{FeO}$, considerando-se razão inicial $\mathrm{Fe}_{2} \mathrm{O}_{3} / \mathrm{FeO}=0,15$.

Sobre os valores $\mathrm{Na}_{2} \mathrm{O}+\mathrm{K}_{2} \mathrm{O}$ versus $\mathrm{SiO}_{2}$, estas rochas situam-se na parte mais rica em álcalis do campo dos picrobasaltos, utilizando-se a grade de classificação proposta por Lê Bas et al. (1986) (Fig. 6). Quando considerados segundo os parâmetros de Wilkinson (1986), correspondem aos picritos alcalinos, posto que a quantidade dos minerais fêmicos é

Tabela 1- Composição modal dos picritos alcalinos Table l- Modal composition of the alkaline picrites superior a 75\%, a composição do plagioclásio normativo é maior que an $50 \%$ e apresentam ne na norma.

A razão $\mathrm{K}_{2} \mathrm{O} / \mathrm{Na}_{2} \mathrm{O}$, dessas rochas, usada para diferenciar as séries sódicas das potássicas, não pode ser aplicada sem que antes seja considerada a possibilidade da incidência de metassomatismo sódico, tal como é exemplificado pela transformação de leucita em analcita. Como já discutido por Danni et al. (1990), para outras rochas da província, este processo de alteração imprime caráter falsamente sódico a rochas originalmente potássicas. Assim, tendo em conta a frequente presença de biotita e feldspato potássico nessas rochas e, desde que se considere estes minerais como expressão heteromórfica do par olivina + leucita (Yoder 1986), os picritos estudados devem ser considerados como ligeira-

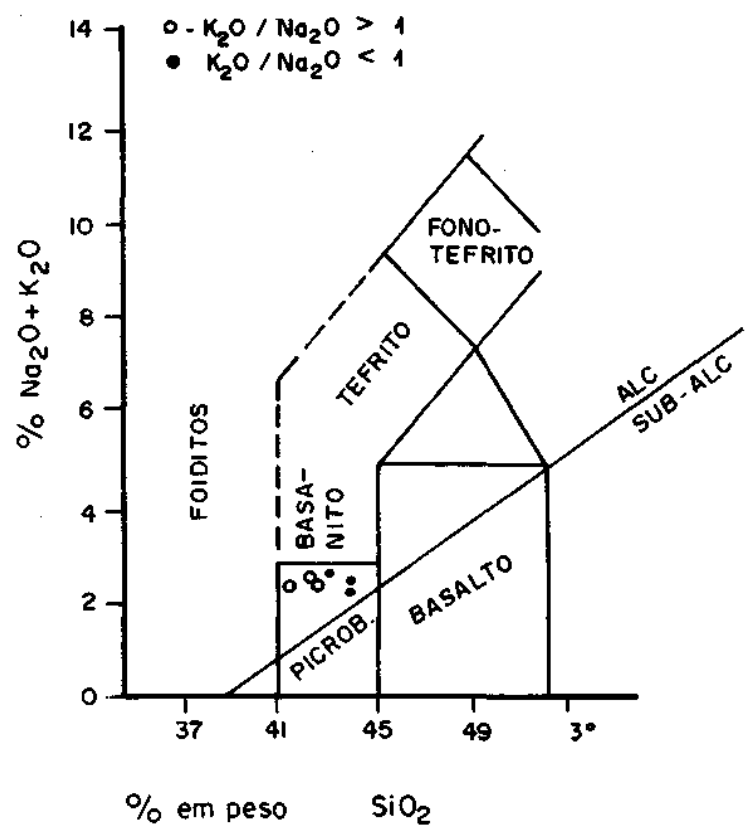

mente potássicos. Do ponto de vista normativo, apresentam

\section{PICRITOS DA REGIÃO DE IPORA'}

Figura 6-Posição dospicritos no diagrama $\mathrm{Na}_{2} \mathrm{O}+\mathrm{K}_{2} \mathrm{Ox}$ $\mathrm{SiO}_{2}$ (Le Bas et al. 1986)

Figure 6 - Position of the picrites in the $\mathrm{NajO}+\mathrm{KjO} \times \mathrm{SiO}_{2}$ diagram (Lê Bas et al. 1986)

\begin{tabular}{l|c|c|c|c|c|c|c|c}
\hline MACROCRISTAIS & \multicolumn{1}{|c|}{$\mathbf{1}$} & 2 & 3 & 4 & 5 & 6 & 7 & Média \\
\hline Olivina-XN & 18,3 & 22,5 & 16,9 & 29,0 & 16,0 & 16,3 & 24,4 & 20,5 \\
Olivina-F & 9,0 & 9,6 & 12,3 & 6,8 & 21,0 & 9,6 & 12,2 & 11,5 \\
Cpx-XN & - & - & - & 7,7 & - & - & - & 1,1 \\
CpX-F & 6,9 & 6,0 & 16,6 & - & 9,5 & 5,4 & 1,0 & 6,3 \\
\hline & & & & & & & & \\
MICROCRISTAIS & & & & & & & & \\
Olivina & 3,1 & 2,3 & 2,5 & - & 3,0 & 5,0 & 0,3 & 2,3 \\
Cpx & 31,5 & 21,7 & 24,0 & 29,1 & 25,6 & 31,4 & 32,6 & 28,0 \\
Espinélio & 13,4 & 5,3 & 5,2 & 6,6 & $-7,7$ & 11,4 & 7,1 & 8,0 \\
Biotita & 2,0 & 9,4 & 4,1 & 3,3 & 4,0 & 1,9 & 1,5 & 3,7 \\
$*$ Min, Félsicos & 15,7 & 24,0 & 18,6 & 17,0 & 12,0 & 19,0 & 20,6 & 18,19 \\
\hline
\end{tabular}

$\mathrm{XN}=$ xenocristais e nódulos; $\mathrm{F}=$ fenocristais; $*=\mathrm{Plag}+\mathrm{K}$ Fel + Nef. + Leuc 
Tabela 2 - Composição química dos picritos alcalinos

Table 2 - Chemical composition of the alkaline picrites

\begin{tabular}{|c|c|c|c|c|c|c|c|c|}
\hline & 1 & 2 & 3 & 4 & 5 & 6 & 7 & 8 \\
\hline$\overline{\mathrm{SiO}_{2}}$ & 42,95 & 43,70 & 43,70 & 41,08 & 42,18 & 42,61 & 42,70 & 44,48 \\
\hline $\mathrm{TiO}_{2}$ & 2,36 & 2,08 & 1,99 & 1,81 & 2,26 & 2,47 & 2,16 & 2,25 \\
\hline $\mathrm{Al}_{2} \mathrm{O}_{3}$ & 8,41 & 9,01 & 6,07 & 6,42 & 8,87 & 6,98 & 7,62 & 7,93 \\
\hline $\mathrm{Fe}_{2} \mathrm{O}_{1}$ & 8,93 & 3,57 & 2,83 & 8,06 & 2,63 & 9,33 & 5,90 & 6,14 \\
\hline $\mathrm{FeO}$ & 4,54 & 8,60 & 8,47 & 7,27 & 8,31 & 2,36 & 6,59 & 6,86 \\
\hline $\mathrm{MnO}$ & 0,15 & 0,15 & 0,15 & 0,16 & 0,18 & 0,16 & 0,16 & 0,17 \\
\hline $\mathrm{MgO}$ & 17,69 & 17,71 & 22,97 & 19,05 & 21,10 & 16,02 & 19,09 & 19,88 \\
\hline $\mathrm{CaO}$ & 9,38 & 9,99 & 8,67 & 8,32 & 9,38 & 9,42 & 9,19 & 9,57 \\
\hline $\mathrm{Na}_{2} \mathrm{O}$ & 1,49 & 1,40 & 1,34 & 0,86 & 1,03 & 0,94 & 1,17 & 1,87 \\
\hline $\mathrm{K}_{2} \mathrm{O}$ & 1,02 & 0,97 & 0,94 & 1,37 & 1,42 & 1,43 & 1,19 & 1,23 \\
\hline $\mathrm{P}_{2} \mathrm{O}_{3}$ & 0,43 & 0,29 & 0,19 & 0,26 & 0,32 & 0,35 & 0,30 & 0,31 \\
\hline P.F. & 2,92 & 2,38 & 2,43 & 5,50 & 1,90 & 7,25 & 3,73 & \\
\hline Total & 100,27 & 99,84 & 99,75 & 100,16 & 99,58 & 99,32 & 99,80 & 100,0 \\
\hline $\mathrm{K} / \mathrm{Na}$ & 0,69 & 0,70 & 0,70 & 1,59 & 1,37 & 1,50 & 1,0 & 1,1 \\
\hline $\mathrm{mg}$ & 0,72 & 0,73 & 0,79 & 0,70 & 0,78 & 0,72 & 0,74 & 0,74 \\
\hline
\end{tabular}

$\mathrm{mg}=\mathrm{MgO} / \mathrm{MgO}+\mathrm{FeO}(\% \mathrm{~mol}), \mathrm{Fe}_{2} \mathrm{O} / \mathrm{FeO}=0,17$

7 = média com P,F; 8 = média na base anidra

Tabela 3 - Norma CIPW dos picritos alcalinos

Table 3 - CIPW Norm of the alkaline picrites

\begin{tabular}{lccccccc}
\hline & 1 & 2 & 3 & 4 & 5 & 6 & Média \\
\hline or & 6,03 & 5,73 & 5,56 & 8,10 & 8,39 & 8,45 & 7,0 \\
ab & 8,26 & 8,07 & 6,54 & 3,72 & 0,33 & 7,95 & 5,8 \\
an & 13,25 & 15,44 & 7,77 & 9,61 & 15,39 & 10,60 & 12,0 \\
ne & 2,35 & 2,05 & 2,60 & 1,93 & 4,54 & - & 2,2 \\
di & 18,66 & 20,12 & 22,51 & 17,81 & 19,20 & 21,10 & 20,2 \\
hd & 5,81 & 5,69 & 4,53 & 6,32 & 3,92 & 6,00 & 5,4 \\
en & - & - & - & - & - & 3,25 & - \\
fs & - & - & - & - & - & 1,06 & - \\
fo & 24,82 & 24,38 & 32,78 & 27,47 & 30,60 & 18,33 & 26,4 \\
fa & 9,77 & 8,72 & 8,35 & 12,34 & 7,91 & 6,77 & 9,0 \\
mt & 2,93 & 2,65 & 2,46 & 3,33 & 2,38 & 2,54 & 2,7 \\
il & 4,48 & 3,95 & 3,78 & 3,44 & 4,29 & 4,69 & 4,1 \\
ap & 1,02 & 0,69 & 0,45 & 0,62 & 0,76 & 0,83 & 0,7 \\
M & 83,4 & 84,1 & 85,4 & 86,3 & 80,1 & 83,6 & 83,8 \\
AN & 61,6 & 65,7 & 55,0 & 72,2 & 97,9 & 57,2 & 68,2 \\
\hline
\end{tabular}

$\mathrm{M}=\%$ Min. ferromagnesianos; $\mathrm{AN}=(\mathrm{an} / \mathrm{ab}+\mathrm{an}), 100$

caráter alcalino insaturado, caracterizado pela presença do par nefelina + olivina. Observa-se, para o conjunto dos picritos analisados, que as variações químicas, quando relacionadas ao índice $\mathrm{MgO} / \mathrm{MgO}+\mathrm{FeO}$, traduzem sobretudo as variações das proporções na acumulação de olivina (Figs. 7 e 8). Dessa forma, as fácies com valores de $\mathrm{MgO} /$ $\mathrm{MgO}+\mathrm{FeO}$ inferiores são as que mais se aproximam da composicão do líquido original. Com respeito aos elementos de transição, os picritos apresentam altas concentrações em Ni (> 550 ppm), Cr (> 520 ppm) e V (>312 ppm), os quais coexistem com valores igualmente elevados de elementos litófilos, como por exemplo $\mathrm{Zr}$ (entre 245 - 168 ppm), Sr (entre 350 - 660 ppm), Ba (entre 350 - 640 ppm). Os elementos terras raras (ETR), quando normalizados para valores condríticos, apresentam um padrão de distribuição retilíneo, com pequena inflexão no Eu e forte inclinação devido ao enriquecimento de ETR leves, quando comparado com os valores de ETR pesados $\left[(\mathrm{La} / \mathrm{Lu})_{\mathrm{n}} 23\right.$ a 29 vezes, Fig. 9]. A distribuição dos elementos traços dessas rochas, quando normalizada a valores de MORB e comparada com 

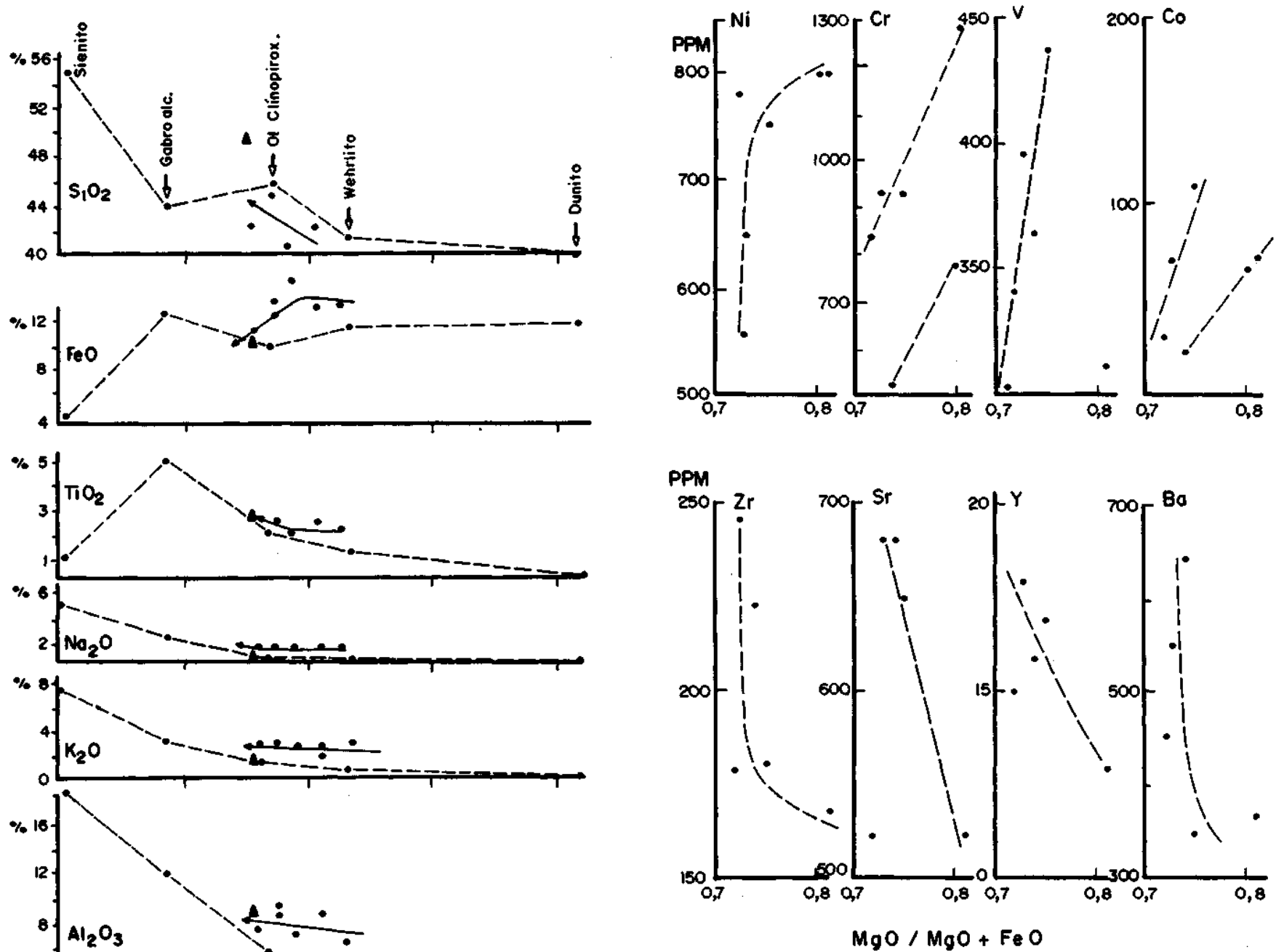

Figura 8 - Diagramas de variação mostrando as variações dos elementos traços versus $\mathrm{MgO} / \mathrm{MgO}+\mathrm{FeO}$ (\% molecular). $\mathrm{O}$ valor de $\mathrm{FeO}$ foi normalizado para $\mathrm{Fe}_{2} \mathrm{O}_{3} / \mathrm{FeO}=0,15$

Figure 8 - Variation diagrams of the trace elements versus $\mathrm{MgO}$ / $\mathrm{MgO}+\mathrm{FeO}$ (molecular \%). The $\mathrm{FeO}$ value was calculated from $\mathrm{Fe}_{2} \mathrm{O}_{3} / \mathrm{FeO}=0,15$

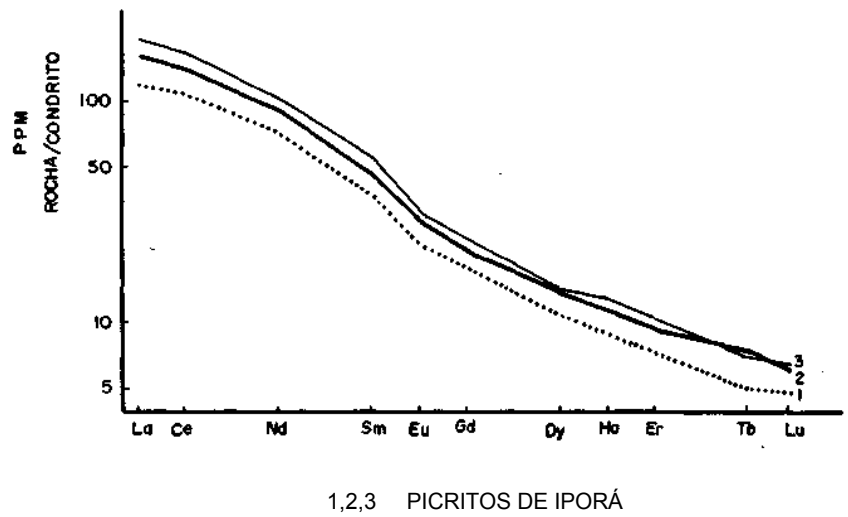

Figura 9 - Padrão de distribuição dos Elementos Terras Raras dos picritos

Figure 9 - Chondrite normalized REE pattern for the analysed picrites

os dos picritos tholeiíticos do Karoo, mostra comportamento análogo, tendo como característica comum a forte anomalia positiva de $\mathrm{Ba}$ (Fig. 10). A anomalia em Ba é uma feição marcante de diversos tipos de magmatismo intracontinental (Wilson 1989) e pode ser atribuída, tanto à presença de flogopita na fonte mantélica como derivada da contaminação crustal. A baixa concentração de ETR pesados parece indicar a ausência de granada na fonte mantélica ou permanência desse mineral no resíduo da fusão. A ausência de

xenolitos mantélicos com granadas na região estudada aponta para a segunda hipótese. Por outro lado, o forte fracionamento de ETR leves pode ser interpretado como resultante da maior participação de clinopiroxênio e, também, de flogopita nos líquidos gerados da fusão mantélica. 
Tabela 4-Elementos traços e de terras raras (ppm) dos picritos

Table 4 - Trace elements and rare earth elements (ppm) of the alkaline picrites

\begin{tabular}{lccccccc}
\hline & 1 & 2 & 3 & 4 & 5 & 6 & Média \\
\hline $\mathrm{Zn}$ & 85 & 80 & 260 & 85 & 83 & 80 & 112 \\
$\mathrm{Co}$ & 110 & 115 & 133 & 135 & 155 & 135 & 130 \\
$\mathrm{Ni}$ & 550 & 780 & 800 & 650 & 750 & 800 & 721 \\
$\mathrm{Cr}$ & 520 & 830 & 770 & 931 & 930 & 1280 & 876 \\
$\mathrm{~V}$ & 364 & 340 & - & 395 & 437 & 312 & 370 \\
$\mathrm{Cu}$ & 88 & 94 & 119 & 89 & 118 & 66 & 95 \\
$\mathrm{Zr}$ & 223 & 179 & 190 & 245 & 180 & 168 & 199 \\
$\mathrm{Y}$ & 16 & 15 & 18 & 18 & 17 & 13 & 15 \\
$\mathrm{Sr}$ & 662 & 350 & 410 & 660 & 600 & 350 & 524 \\
$\mathrm{Ba}$ & 643 & 450 & 570 & 550 & 350 & 370 & 472 \\
$\mathrm{Rb}$ & - & - & 29 & 26 & - & 24 & 26 \\
$\mathrm{Nb}$ & - & - & 36 & 38 & - & 26 & 33 \\
$\mathrm{Th}$ & - & - & 15 & 14 & - & 13 & 14 \\
$\mathrm{Hf}$ & - & - & 23 & 30 & - & 43 & 32 \\
$\mathrm{La}$ & - & - & 38,8 & 46,4 & - & 30, & 38,4 \\
$\mathrm{Ce}$ & - & - & 93,8 & 108,5 & - & 74,0 & 92,0 \\
$\mathrm{Nd}$ & - & - & 43,7 & 49,5 & - & 34,1 & 42,4 \\
$\mathrm{Sn}$ & - & - & 7,5 & 8,3 & - & 5,86 & 7,2 \\
$\mathrm{Eu}$ & - & - & 1,67 & 1,87 & - & 1,35 & 1,63 \\
$\mathrm{Gd}$ & - & - & 4,61 & 5,19 & - & 3,82 & 4,54 \\
$\mathrm{Dy}$ & - & - & 3,60 & 3,74 & - & 2,83 & 3,30 \\
$\mathrm{Ho}$ & - & - & 0,69 & 0,72 & - & 0,54 & 0,65 \\
$\mathrm{Er}$ & - & - & 1,70 & 1,78 & - & 1,31 & 1,60 \\
$\mathrm{Yb}$ & - & - & 1,26 & 1,24 & - & 0,92 & 1,14 \\
$\mathrm{Lu}$ & - & - & 0,16 & 0,16 & - & 0,13 & 0,15 \\
\hline
\end{tabular}

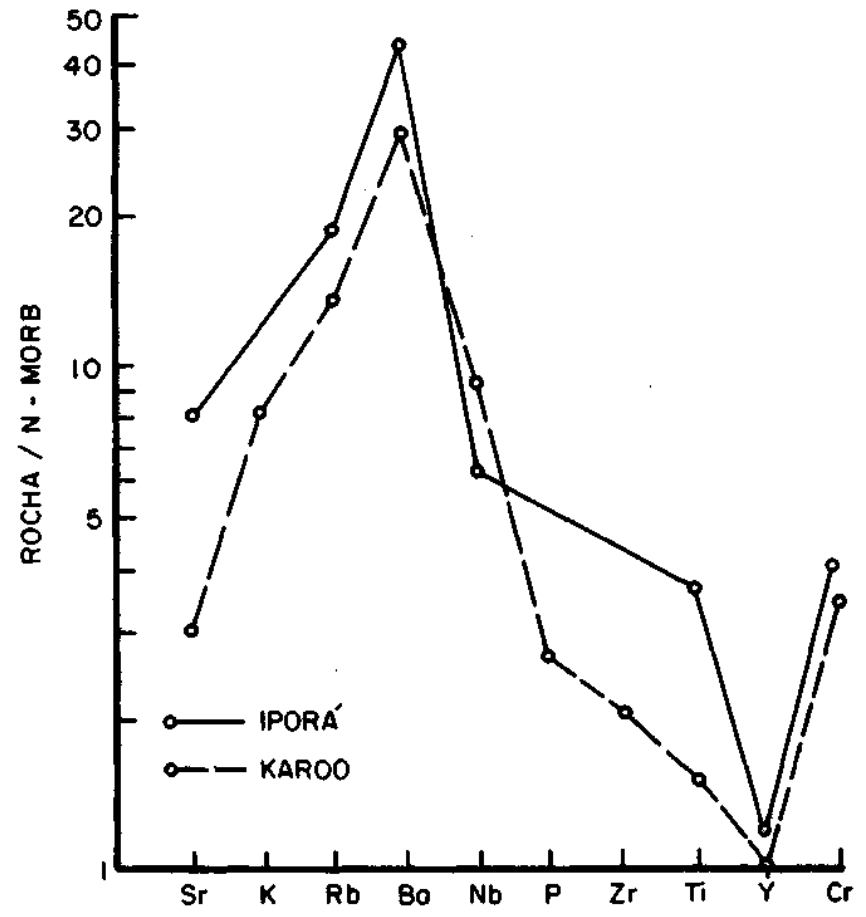

VALORES DE N-MORB EM BRISTOW, 1984

Figura 10-Distribuição de elementos litófilos e de transição dos picritos de Iporá, comparado com picritos do Karoo, ambos normalizados a valores de $N$-MORB

Figure 10 - Comparison of the distribution of the LIL and HFS elements plus $\mathrm{Cr}$ betweenn the lpora and Karoo picrites
Tabela 5 - 1. Composição química média de seis picritos analisados; 2 e 3. Composicões químicas derivadas por subtração de 20 e $15 \%$ de olivina ( $F o_{\text {s }}$ ) a partir de $I$, respectivamente

Table 5 - 1. Chemical composition of six samples of picrites; 2 . and 3. Chemical composition calculated by subtraction of $20 \%$ and $15 \%$ of olivine $\left(\mathrm{Fo}_{38}\right.$ ) from compositon 1 , respectively

\begin{tabular}{lccc}
\hline & 1 & 2 & 3 \\
\hline $\mathrm{SiO}_{2}$ & 44,48 & 45,43 & 45,15 \\
$\mathrm{TiO}_{2}$ & 2,25 & 2,81 & 2,65 \\
$\mathrm{Al}_{2} \mathrm{O}_{3}$ & 7,93 & 9,91 & 9,33 \\
$\mathrm{Fe}_{2} \mathrm{O}$, & 1,61 & 1,65 & 1,78 \\
$\mathrm{FeO}$ & 10,76 & 9,90 & 10,73 \\
$\mathrm{MnO}$ & 0,17 & 0,15 & 0,16 \\
$\mathrm{MgO}$ & 19,88 & 13,32 & 15,25 \\
$\mathrm{CaO}$ & 9,57 & 11,83 & 11,16 \\
$\mathrm{Na}, \mathrm{O}$ & 1,21 & 1,51 & 1,42 \\
$\mathrm{~K}_{2} \mathrm{O}$ & 1,23 & 1,54 & 1,45 \\
$\mathrm{PO}_{2} \mathrm{O}$ & 0,31 & 0,39 & 0,36 \\
$\mathrm{mg}$ & 0,76 & 0,70 & 0,71 \\
\hline
\end{tabular}

$\mathrm{mg}=\mathrm{MgO} / \mathrm{MgO}+\mathrm{FeO}(\% \mathrm{~mol}) ; \mathrm{Fe}_{2} \mathrm{O}_{3} / \mathrm{FeO}=0,15$ 
Ainda que as características químicas sejam indicativas de origem primária para os líquidos precursores desses picritos, deve ser considerada a influência da acumulação de olivina. Isto é bem evidente se considerados os altos valores da razão $\mathrm{MgO} / \mathrm{MgO}+\mathrm{FeO}$, apresentados por essas rochas, em nítido desequilíbrio com o teor da molécula de Fo das olivinas (Fo $87-88 \%$ ), desde que se considere $\mathrm{Kd}=0,30$ (Roeder e Emslie 1970) e a razão $\mathrm{Fe}_{2} \mathrm{O}_{3} / \mathrm{FeO}=0,15$. Para corrigir o desvio composicional dos líquidos gerados pela acumulação de olivina, foram realizados cálculos de subtração pelo programa de Stormer e Nicholls (1978), tendo por base a quantidade e o teor de forsterita dos fenocristais encontrados nessas rochas. Dessa forma, obtiveram-se composições de líquidos com razões de $\mathrm{MgO} / \mathrm{MgO}+\mathrm{FeO}$ entre $0,70-0,71$, cujos resultados globais encontram-se na tabela 3 . É provável que a composição $n^{9} 3$ da tabela 5 represente a composição mais apropriada para o magma parental. Nessa hipótese, considera-se que parte dos cristais de olivina, não subtraídos no cálculo de massa, possa representar fases em equilíbrio com o líquido, gerados pelo processo de cristalização fracionada compensada, como proposto por Cox et al. (1984).
CONCLUSÕES Na Província Alcalina do sul de Goiás, é definida a ocorrência de intrusões subvulcânicas constituídas de picritos alcalinos. Essas rochas possuem texturas porfiríticas cumuláticas, formadas por macrocristais de olivina $\left(\mathrm{Fo}_{88}\right)$. Esporadicamente, ocorrem xenólitos de dunitos e olivina clinopiroxenitos, em tudo análogos aos que formam os complexos plutônicos da região, evidenciando estreita relação genética entre ambos.

Por meio de cálculos de massa por subtração das frações de olivina cumulus dos picritos, pode-se derivar composições de basaltos picríticos alcalinos com 13,0\% de $\mathrm{MgO}$ e $\mathrm{MgO} / \mathrm{MgO}+\mathrm{FeO}=0,70$. Postula-se que tais composições representem líquidos parentais que deram origem, por cristalização fracionada, às séries de rochas que constituem os complexos plutônicos da região de Iporá.

Agradecimentos $\mathrm{O}$ Autor expressa seus agradecimentos a A.J.G.C. Silva, J. A. Brod, J.E. Campos, M. Cerqueira e G. Boaventura pela colaboração prestada na obtenção de dados. Ao CNPq (Proc. $n^{9}$ 300899/82-3/GL-FV) pelo apoio financeiro.

\section{REFERÊNCIAS BIBLIOGRÁFICAS}

BARBOUR, A.P. 1976. Geologia do Maciço Ultramáfico de Santa Fé, Goiás. São Paulo. 138 p. (Tese de Livre Docência, IG-USP).

BRISTOW, J.W. 1984. Picritic rocks of the north Lebombo and South-east Zimbabwe. Geol Soe. S. Afr. Trans., Spec. Publ, 13:105-123.

CLARKE, D.B. \& O'HARA, MJ. 1979. Nickel and the existence of high$\mathrm{MgO}$ liquids in nature. Earth Planei. Sei. Lett., 44:153-158

COX, K.G. 1987. Postulated restite fragments from Karoo picrite basalts: their bearing on magma segregation and mantle deformation. J. Geol. Soe. London, 144:275-280.

COX, K.G.; DUNCAN, A.R.; BRISTOW, J.W.; TAYLOR, S.R.; ERLANK, A.J. 1984. Petrogenesis of the basic rocks of the Lebombo Geol Soe. S. Afr. Trans., Spec. Publ., 13:149-169.

DANNI, J.C.M. 1974. Geologie dês Complexes Ultrabasiques Alcalins de Ia Region d'Iporá - GO. Orsay, França. 95 p. (These Doctotat, Universite Paris Sud).

DANNI, J.C.M. 1976. Magmatic differentiation of the alkaline-ultrabasic intrusions of the Iporá region, South-West Goiás, Brazil, In: INTERN. SYMP. CARBONATITES. Poços de Caldas, 1976. Actas.. Poços de Caldas, MME-DNPM/IACG. p. 149-167.

DANNI, J.C.M. 1985. Rochas da Série Kamafugitica na Região de Amorinópolis, Goiás. Belo Horizonte, SBG/MMG. p. 5-13. (Boletim Especial).

DANNI, J.C.M.; SILVA, A.J.; CERQUEIRA, M.R. 1990. Petrografia e petroquímica das rochas alcalinas cretácicas da Serra do Caiapó, SW de Goiás. In: CONGR. BRÁS. GEOL., 36. Natal, 1990. Anais... Natal, SBG. v. 4, p. 1872-1882.

FARIA, A.; RIPPLE, C.; GLASER, J.; BEZ, L.; MARQUES, VJ 1970 Mapa geológico da região de Iporá, GO. In: CONGR. BRAS. GEOL., 21. São Paulo, 1970. Boletim de Resumos... São Paulo, SBG. p. 120-121.

FODOR, R.V. 1987. Low and high-TiO ${ }_{2}$ flood basalts of the Southern Brazil: origin from picritic parentage and a common mantle Source. Earth Planei. Sei. Lett., 86:423-430.

FODOR, R.V.; MUKASA, S.B.; GOMES, C.B.; CORDANI, U.U. 1989. Ti-rich Eocene basaltic rocks, Abrolhos Platform, offshore Brazil, $18^{\circ} \mathrm{S}$ : Petrology with respect to South Atlantic Magmatism./. Petrol, $3<\mathrm{K} 3): 763-786$

FOSTER, M.D. 1960. Interpretation of the composition of trioctahedral micas. Washington, U. S. Geol. Surv. Prof. Paper, 354-B:48 p.

FROST, B.R. \& LINDSLEY, D.H. 1992. Occurence of iron-titanium oxides in igneous rocks, in Oxides Minerais. Am. Mineral., 12:434-467. (Spec. Issue).

GASPAR, J.C. \& DANNI, J.C.M. 1981. Aspectos petrográficos e vulcanológicos da Província Alcalina-Carbonatitica de Santo António da Barra, SW de Goiás. Rev. Brás. Geoc., 11:78-83.

GUIMARÃES, G.; GLASER, L; MARQUES, VJ. 1968. Sobre a ocorrência de rochas alcalinas na região de Iporá-Goiás. Min. Metal., 448(283): 11-15.

HASUI, Y.; DYER, R.C.; IWANUCH, W. 1971. Geocronologia das rochas alcalinas de Santo António da Barra, GO. In: CONGR. BRÁS. GEOL., 21. São Paulo, 1971. Anais... SBG, São Paulo. v. 1, p. 253-258.
Lê B AS, M.J. 1989. Nephelinitic and basanitic rocks. J. Petrol., 30:1299-1312.

Lê BAS, JJ.; Lê MAITRE, R.W.; STRECKEISEN, A.; ZANETINN, B. 1986. A chemical classification of volcanic rocks based on the total alkali-silica diagram. J. Petrol., 27(3):745-750.

NICHOLLS, J. \& STOUT, MZ. 1988. Picritic melts in Kilauea-Evidence from the 1967-1968 Halemauman and Hiiaka Eruptions. J. Petrol., 29(5):103M057.

PENA, G.S. \& FIGUEIREDO, MJ. 1972. Projeto Alcalinas. Goiânia, DNPM-CPRM. 143 p. (Relatório Inédito).

PICCIRILLO E.M \& MELFI, A.J. 1988. The Mesozoic Flood Volcanism of the Paraná Basin - Petrogeneíic and Geophysical Aspecís. São Paulo, IAG-USP. $600 \mathrm{p}$

PICCIRILLO, E.M.; BELLIENI, G.; COMIN-CHIARAMONTI, P.; ERNESTO, M.; MELFI, A.J.; PACCA, J.G.; USSAMI, N. 1988. Significance of the Paraná flood volcanism in the disruption of western gondwanaland. In: PICCIRILLO, E.M. \& MELFI, A J. ed. The Mesozoic Flood Volcanism of the Paraná Basin. São Paulo, IAG-USP. p. 287-295.

PICCIRILLO, E.M.: COMIN-CHIARAMONTI, R; BELLIENI, G.; CIVETTA, L; MARQUES, L.S.; MELFI, A J.; PETRINI, R.; RAPOSO, M.B.; STOLFA, D. 1988. Petrogenetic aspects of continental flood basalts-rhyolite suites from the Paraná Basin (Brazil). In: PICCIRILLO, E.M. \& MELFI, A.J. ed. The Mesozoic Flood Volcanism ofthe Paraná Basin. São Paulo, IAG-USP. p. 179-205.

PIMENTEL, M. \& FUCK, R.A. 1987. Late Proterozoic Magmatism in Southweestern Goiás, Brazil. Rev. Brás. Geoc., 17(4):415-425.

ROEDER, P.L. \& EMSLIE, R.F. 1970. Olivine-liquid equilibrium. Contrib. Mineral. Petrol., 29:275-289.

STORMER, J.C. \& NICHOLLS, J. 1978. XLFRAC; A program for the interactive testing of magmatic differentiation models. Comput. \& GeoscL, 4:143-159.

VIERO, A.P. 1992. Petrologia e Geoquímica do Complexo Básico de Lomba Grande, R.S. Porto Alegre. 176 p. (Dissertação de Mestrado, IG-UFRGS)

WILKINSON, J.F.G. 1986. Classification and average chemical compositions of common basalts and andesites. J. Petrol., 27(1):31-62.

WILKINSON, J.F.G. \& HENSEL, H.D. 1988. The petrology of some picrites from Mauna Loa and Kilauea volcanoes, Hawaii. Contrib. Mineral. Petrol., 98:326-345.

WILSON, M. 1989. Igneous Petrogenesis. London, Unwin Hyman Press. $466 \mathrm{p}$.

YODER, H.S. 1986. Potassium-rich rocks: phase analysis and heteromorphic relations. J. Petrol., 27(5): 1215-1228.

MANUSCRITO A805

Recebido em 22 de novembro de 1993 Revisão do autor em 14 de abril de 1994 Revisão aceita em 28 de abril de 1994 